\title{
EDITORIAL
}

\section{Is Oral Verrucous Hyperplasia a Distinct Entity or a Precursor of Oral Verrucous Carcinoma?}

\author{
${ }^{1}$ A Thirumal Raj, ${ }^{2}$ Shankargouda Patil, ${ }^{3}$ Sachin C Sarode, ${ }^{4}$ Gargi S Sarode
}

How to cite this article: Raj AT, Patil S, Sarode SC, Sarode GS. Is Oral Verrucous Hyperplasia a Distinct Entity or a Precursor of Oral Verrucous Carcinoma? World J Dent 2017;8(4):247.

\section{Source of support: Nil}

Conflict of interest: None

Oral verrucous hyperplasia $(\mathrm{OVH})$ has long been considered to be a precursor of oral verrucous carcinoma (OVC). ${ }^{1}$ Evidence for the precursor hypothesis stems from their similar clinicopathological presentations. Both $\mathrm{OVH}$ and OVC may present clinically as an exophytic lesion with surface projections (blunt or sharp) imparting a verrucous appearance. Both the lesions are strongly associated with tobacco usage and share similar demographics including a male predominance, affinity to buccal mucosa, and peak prevalence above the 5 th decade of life. ${ }^{2}$ Both $\mathrm{OVH}$ and OVC are mostly solitary, but multiple presentations have been reported especially as a part of oral proliferative verrucous leukoplakia.

The histopathological similarities of OVH and OVC include a hyperkeratinized surface epithelium with multiple exophytic projections and characteristic keratin pluggings. Both the lesions can also exhibit epithelial dysplasia (ED) and subepithelial inflammatory reaction. Multiple endophytic and exophytic growths are noted in both $\mathrm{OVH}$ and OVC. ${ }^{2}$

Although the above-mentioned clinicopathological similarity strengthens the precursor hypothesis, there are unique histopathological features that distinguish OVH from OVC. These histopathological features can be categorized as major and minor based on the degree of the uniqueness of the feature to a lesion.

\footnotetext{
${ }^{1}$ Department of Oral Pathology and Microbiology, Sri Venkateswara Dental College and Hospital, Chennai, Tamil Nadu, India

${ }^{2}$ Division of Oral Pathology, Department of Diagnostic Sciences College of Dentistry, Jazan University, Jazan, Kingdom of Saudi Arabia

${ }^{3,4}$ Department of Oral Pathology and Microbiology, Dr. D. Y. Patil Dental College \& Hospital, Dr. D. Y. Patil Vidyapeeth, Pune Maharashtra, India

Corresponding Author: A Thirumal Raj, No.53, C-6 Block Todhunter Nagar, Saidapet, Chennai, Tamil Nadu, India, Phone: +918122627810, e-mail: id:thirumalraj666@gmail.com
}

The sole major feature is the presence or absence of an endophytic growth of the lesional epithelium below the level of the basement membrane of the adjacent normal epithelium. Thus, although both OVH and OVC may present exophytic and endophytic growth, it is vital to note the extent of the endophytic growth. If the endophytic growth of the lesional epithelium is below the level of the basement membrane of the adjacent normal epithelium, the diagnosis is of OVC and if the lesional epithelium has endophytic growth but it is either above or at the level of the basement membrane of the adjacent normal epithelium, the diagnosis is of $\mathrm{OVH}^{2}{ }^{2}$ The minor feature includes the presence or absence of ED. Although the epithelium of both the lesions can exhibit dysplastic features, its prevalence is significantly more in OVH. The increased prevalence of dysplasia in OVH over OVC, in turn, is considered as significant evidence to suggest $\mathrm{OVH}$ is not a precursor to OVC. The basis for the above statement stems from the fact that dysplasia is a key feature indicating progression into malignancy. Thus, for an $\mathrm{OVH}$ with dysplasia to transform into an OVC without dysplasia, the OVH must lose its dysplastic features, which is against the norms of malignant transformation. ${ }^{3}$

To conclude, conclusive data on the natural history of $\mathrm{OVH}$ requires the molecular characterization of histopathologically diagnosed cases of $\mathrm{OVH}$ with dysplasia, $\mathrm{OVH}$ without dysplasia, OVC with dysplasia, and OVC without dysplasia. Until then, it can be hypothesized that $\mathrm{OVH}$ without dysplasia may progress to either OVC or directly to oral squamous cell carcinoma (OSCC) and OVH with dysplasia is more likely to progress directly to OSCC. Surgeons should keep in mind that the final diagnosis of OVC requires an adequate sampling of the tissues. Due to lack of the dysplastic features in the individual cells, the pathologist must carefully appraise the complete histomorphologic configuration of the lesion to arrive at a correct diagnosis. Sufficient sampling also is imperative because as many as $20 \%$ of these lesions have a routine squamous cell carcinoma developing concurrently within the verrucous carcinoma.

\section{REFERENCES}

1. Slootweg PJ, Müller H. Verrucous hyperplasia or verrucous carcinoma: an analysis of 27 patients. J Maxillofac Surg 1983 Feb;11(1):13-19.

2. Patil S, Warnakulasuriya S, Raj T, Sanketh DS, Rao RS. Exophytic oral verrucous hyperplasia: a new entity. J Investig Clin Dent 2016 Nov;7(4):417-423.

3. Raj AT. Oral lichenoid dysplasia and exophytic oral verrucous hyperplasia: mythical entities of oral pathology. Oral Oncol 2017 Jul;70:75. 